\title{
Maren Goltz
}

\section{»Den Käuffern zur Danksagung vndt zur recorirung des Gemüths« - Zur Funktion der Musik auf den Bühnen der »ciarlatani «}

Als Fynes Moryson Anfang des 17. Jahrhunderts die Eindrücke seiner Italienreisen Revue passieren ließ, bemerkte er in Bezug auf das im Italien des 16. und frühen 17. Jahrhunderts allgegenwärtige Phänomen der ciarlatani folgendes:

Also not only in Carnavall but all the yeare long, all the Markett places of great Cittyes are full of Montebanks, or Ciarlatanes, who stand upon tables like stages, and sell their oyles, waters and salves, drawe the people about them by musicke and pleasant discourse like comedies, having a woman and a masked fool to acte these partes with them. ${ }^{2}$

Nach Moryson verstanden es die ciarlatani also, während des bunten Markttreibens in den Städten durch den Einsatz von Musik und Komödianten Aufmerksamkeit zu erregen und Marktbesucher anzulocken, um ihre Salben, Tinkturen und dergleichen zu verkaufen.

Zunächst ein paar kurze Bemerkungen vorweg zum Begriff des ciarlatano. Abgeleitet vom italienischen ciarlare (gewandt reden, mit Untertönen des Schmeichelns und große Reden-Schwingens) bezeichnet er nach Burke »im wesentlichen Leute ohne ärztlichen Titel, die Arzneien - und manchmal andere Artikel öffentlich, vor einem Publikum verkauften «. ${ }^{3}$ Synonyme begegnen selbstverständlich auch in anderen Sprachen, im 16. Jahrhundert abgeleitet vom niederländischen kvakzalver etwa der deutsche Quacksalber. Anfang des 17. Jahrhunderts kennt man in Deutschland auch die Ableitung vom italienischen ciarla-

1 Das Referat ist die überarbeitete Fassung eines Vortrages, den ich bereits in den Jahren 1997 und 1999 gehalten habe, in Leipzig vor einem in erster Linie theaterhistorisch interessierten, in Como di Menaggio vor einem in erster Linie medizinhistorisch interessierten Publikum. Frau Prof. Dr. Nicole Schwindt sei an dieser Stelle nochmals für die Einladung zum Symposium gedankt, die neben der Herausforderung eines musikwissenschaftlichen Zusammenhangs erstmals eine Konzentration der Fragestellungen auf die Zeit der Renaissance ermöglichte. Sehr herzlich danke ich zudem Frau Dr. Birgit Heise vom Musikinstrumentenmuseum der Universität Leipzig für ihre Hinweise.

2 Shakespeare's Europe. Unpublished Chapters of Fynes Moryson's Itinerary, being a Survey of the Condition of Europe at the End of the $16^{t h}$ Century, hrsg. von Charles Hughes, London 1903, S. 465.

3 Peter Burke, Städtische Kultur in Italien zwischen Hochrenaissance und Barock. Eine bistorische Anthropologie, Berlin 1986, S. 178f. 
tano: den Scharlatan, in Frankreich den charletan. Um die mit den deutschen Begriffen Quacksalber und Scharlatan etwa seit dem 17. Jahrhundert verbundene negative Konnotation ${ }^{4}$ zu vermeiden, argumentiere ich im Folgenden mit dem italienischen Begriff des ciarlatano.

Doch zurück zur Beschreibung von Fynes Moryson. Dass Musik und komödiantisches Spiel offenbar eine zentrale Rolle beim Auftritt der ciarlatani spielten, bezeugen neben Moryson auch andere Quellen vom 16. bis zum 18. Jahrhundert, und zwar nicht nur für Italien, sondern auch für Frankreich und Deutschland. Auftritte musizierender und spielender ciarlatani waren in Europa weit verbreitet und gehörten zum festen Bestandteil der Alltagskultur der Frühen Neuzeit.

Umso mehr verwundert es daher, dass die Forschung diesem Phänomen bisher kaum Beachtung geschenkt hat. Von der älteren medizinhistorischen und theaterwissenschaftlichen Forschung wurde es bisher, mit seltenen Ausnahmen, verächtlich als wenig beachtenswerte Vorform des ehrwürdigen Gegenstandes des jeweiligen Faches eingeordnet, die es alsbald zu überwinden galt. Erst in jüngster Zeit widmet ihm die Theater- und Kulturwissenschaft im Zuge des Forschungsinteresses an der commedia dell'arte etwas mehr Aufmerksamkeit.

Wissenschaftliche Studien zur Problematik der Musik beim Auftritt der ambulanten Ärzte und Heilmittelhändler sucht man freilich vergeblich. Und das, obwohl Percy A. Scholes bereits im Jahre 1938 daran erinnerte, "that a great part of the musical life of the populations of European countries has been lived not in the concert-hall, opera-house, or church, but in the streets «, ${ }^{5}$ und damit quasi ex negativo anmahnte, diesem »often overlooked fact« mehr Aufmerksamkeit zu schenken.

In Ermangelung detaillierter Untersuchungen behilft sich die Forschung, sei es die Kulturwissenschaft oder auch die Medizingeschichte, daher mit Allgemeinplätzen. Meist beschränkt man sich auf die grundsätzliche Feststellung, dass Musik einen wesentlichen Bestandteil des Auftrittes von ciarlatani bildete und zur Anlockung des Publikums bzw. zur Ablenkung vom Leiden der Patienten diente. ${ }^{6}$ So schreibt Peter Burke in seiner Studie Städtische Kultur in Italien

4 Siehe dazu die Einträge "Quacksalber « und "Scharlatan«, in: Etymologisches Wörterbuch des Deutschen, hrsg. von Wolfgang Pfeifer, ${ }^{2}$ Berlin 1993, Bd. 2, S. 1063 f. und 1182.

5 Percy A. Scholes, The Oxford Companion to Music, rev. von John Owen Ward, ${ }^{10}$ London 1978, S. 987.

6 P. Burke, Städtische Kultur (wie Anm. 3), S. 178. Siehe weiterhin Robert Jütte, Ärzte, Heiler und Patienten. Medizinischer Alltag in der frïhen Neuzeit, München 1991, S. 29; Chansons de Gaultier Garguille, hrsg. von Édouard Fournier, Paris 1858, S. liv; Robert Wustmann, Musikgeschichte Leipzigs in drei Bänden, Bd. 1: Bis zur Mitte des 17. Jahrhunderts, Leipzig 1909, S. 76. 
zwischen Hochrenaissance und Barock beispielsweise: "Musik hatte auch ihren Platz in den Aufführungen der bekanntesten italienischen Heilkundigen der Zeit, der sogenannten $>$ Scharlatane $«{ }^{7}$ Weiter unten heißt es: »Sie begleiteten den Verkauf mit einer Art Vorfuihrung, um die Aufmerksamkeit der Zuschauer auf sich zu ziehen. ${ }^{8}$

Johann Friedrich Fischer geht in seinem Aufsatz zum Arztständlspiel im Lande Salzburg erheblich weiter. Bei ihm ist zu lesen: »Auf dieser Bühne wurde gespielt wie operiert, die Lazzi des Spaßmachers mußten vom Operateur und seinem Opfer ablenken, der Lärm eines tollen, wirbelnden Geschehens, der Tanz und die Musik die Schmerzenschreie, das Gestöhn und Ächzen der Patienten übertönen."

\section{Quellenlage}

Bereits ein flüchtiger Blick auf das relevante Quellenmaterial zeigt, dass derartige Erklärungsmuster, die Anlockung von Publikum und die Ablenkung der Patienten, höchstens Teilaspekte des Phänomens erfassen. Erwähnt sei an dieser Stelle bereits der Auftritt von Claudius de Aquaviva, der 1622 in Frankfurt am Main ausdrücklich nach dem Verkauf von Heilmitteln musiziert.

Die Vielschichtigkeit und Komplexität der Thematik erfordert eine systematische Analyse, die nicht nur auf einer breiteren Quellenbasis aufbaut und stärker regional und zeitlich differenziert, sondern vor allem ein umfassenderes Untersuchungsraster zugrunde legt. Denn zunächst bedürfen so grundsätzliche Fragen, wie »Wer spielte wann, auf welchen Instrumenten und auf welche Art und Weise Musik? «, einer Antwort, um Erkenntnisse über die Funktion und die Wirksamkeit der Musik beim Auftritt von ciarlatani zu gewinnen.

Die folgenden Ausfïhrungen erheben selbstverständlich nicht den Anspruch, die erwähnten Forderungen vollständig einzulösen oder gar den Sachverhalt erschöpfend zu klären. Doch soll anhand ausgewählter Quellen, die einen Querschnitt durch das zur Verfuigung stehende gedruckte Quellenmaterial bilden, gezeigt werden, welche vielversprechenden Möglichkeiten, aber auch welche Grenzen des Erkenntnisgewinns sich bei einer systematischen Untersuchung ergeben. Damit sollen Denkräume eröffnet und Anstöße zu weiteren Überlegungen gegeben werden.

P. Burke, Städtische Kultur (wie Anm. 3), S. 178.

Ebda., S. 179.

Friedrich Johann Fischer, „Das Arztständlspiel im Lande Salzburg. Ein Beitrag zur Entstehung und zum Werdegang des deutschen Theaters wie zur Kulturgeschichte Salzburgs im 17. und 18. Jahrhundert«, in: Mitteilungen der Gesellschaft fiir Salzburger Landeskunde 102 (1962), S. 215. 


\section{Methodisches Vorgehen}

Das methodische Herangehen an die Problematik der musizierenden ciarlatani ist in der Tat mit einigen Schwierigkeiten verbunden. Die Annäherung muss gewissermaßen aus verschiedenen Richtungen erfolgen, d.h. über die bildlichen und schriftlichen Quellen $\mathrm{zu}$ musizierenden ciarlatani, die Verbindung des Phänomens zur commedia dell'arte ${ }^{10}$ sowie über die allgemeine Wirksamkeit von Musik und musikalischem Theater.

Die schriftlichen Quellen setzen sich überwiegend aus Aktenvermerken und zeitgenössischen Beschreibungen, etwa von Reisenden, zusammen. Die bisherigen Zeugnisse beziehen sich fast ausnahmslos auf Auftritte von ciarlatani in großen Städten, nicht in ländlichen Gegenden, und dürfen daher auch nur für diesen Bereich Aussagekraft beanspruchen. Dass Unterschiede in der skünstlerischen Preislage bestanden, bezeugt etwa Thomas Coryate, der im Jahre 1611 berichtet, dass die venezianischen ciarlatani als Koryphäen ihres Fachs galten. ${ }^{11}$

Es ist anzunehmen, dass Anzahl und Können der Musiker in der Stadt höher waren, dass man hier schneller auf Moden reagierte und das musikalische Auftreten den Bedingungen eines im Unterschied zum Land größeren und sozial anders gelagerten Marktbetriebes anpasste. Allerdings fehlt hierzu bisher das Vergleichsmaterial, so dass man nicht über Vermutungen hinausgelangt, zumal mit zahlreichen Nuancierungen zu rechnen ist.

Belege für eine nicht näher beschriebene musikalische Tätigkeit von ciarlatani in der Stadt bieten neben Fynes Morysons ${ }^{12}$ und Giovanni Domenico Ottonellis Beobachtungen in Italien ${ }^{13}$ die Akten im Fall des Claudius de Aquaviva (Köln 1621 und Frankfurt am Main 1622) ${ }^{14}$ sowie des Johann Baptista Montedor (Frankfurt am Main 1625). ${ }^{15}$

$10 \mathrm{Zu}$ dieser Problematik siehe Siro Ferrone, Attori mercanti corsari. La Commedia dell'Arte in Europa tra Cinque e Seicento, Turin 1993 (Saggi, 773), S. 50-88.

11 Thomas Coryate, Coryats Crudities 1611, London 1978, S. 272.

12 Vgl. das Tagebuch eines anonym gebliebenen Engländers von einer Italienreise (GB-Lbl, Ms. Sloane 682, fol. 19', 13 May 1610), siehe dazu Margret A. Katritzky, "Was Commedia dell'arte performed by Mountebanks? Album amicorum illustrations and Thomas Platter's description of 1598«, in: Theatre Research International 23 (1998), S. 118.

13 Giovanni Domenico Ottonelli, Della christiana moderatione del theatro, Florenz 1652, S. 456, zit. n. Roberto Tessari, La Commedia dell'Arte nel Seicento. "Industria" $e$ sarte giocosa" della civilità barocca, Florenz 1969, S. 38.

14 Eintrag vom 13. August 1621, Köln: "Medicus Romanus, Jean Potagie-Claudius de Aquaviva Romanus hat bewilligung erhalten, seine medicamenta an offenem Marckt mit agierung etlicher Dialogen und vorhergehender music ein zeitlang zu verkaufen und solches sine scandalo mit dem beding, dass er Sontags und heilig tags damit einhalten solle«, zit. n. Carl Niessen, Drama- 
Mehrere Quellen bezeugen, dass gesungen wurde, so etwa Tomaso Garzoni in seiner Piazza universale aus dem Jahre $1585 .{ }^{16}$ Unter anderem Thomas Coryates Schilderung der ciarlatani auf dem Markusplatz in Venedig aus dem Jahre 1611 ist zu entnehmen, dass selbige sowohl vokale als auch instrumentale Musik praktiziert haben. ${ }^{17}$ Zwei Quellen benennen Instrumente auf den Bühnen der ciarlatani: Garzoni nennt eine »lira«, ${ }^{18}$ Thomas Platter der Jüngere berichtet am Ende des Jahres 1598 von Violen, Lauten und Harfen. ${ }^{19}$ In der ersten deutschen Ausgabe der Piazza universale aus dem Jahre 1619 ist im 104. Diskurs zu lesen:

tische Darstellungen in Köln von 1526-1700, Köln 1917 (Veröffentlichungen des Kölnischen Geschichtsvereins, 3), S. 90. Raths-Supplikationen Eintrag vom April 1622, Frankfurt am Main, darüber, dass Aquaviva in der Ostermesse 1622 "nach dem Absatz und dem Genuss der Hülfsmittel [...] den Käufern zur Danksagung vndt zur recorirung des Gemüths eine liebliche Musika und Comedia nach Art der alten Römer auf italienisch abhielt", zit. n. Elisabeth Mentzel, Geschichte der Schauspielkunst in Frankfurt am Main. Von ibren ersten Anfägen bis zur Eröffnung des städtischen Komödienhauses. Ein Beitrag zur deutschen Kultur-und Theatergeschichte, Frankfurt/M. 1882, S. 62, und C. Niessen, Dramatische Darstellungen (wie oben), S. 91. Ratsdekret von 1622, Frankfurt am Main: »Als Claudius D'Aquaviva, ein Medicus, gebetten, Jhme Zuverstatten, daß er seine Artzneÿ offentlich verkauffen vnd dabey eine Music vnd Jtalianische Comoedien halten möge. Soll man Jhm sein begeren abschlag(en)《, zit. n. Hans Richard Purschke, Puppenspiel und verwandte Künste in der Freien Reichs-Stadt Frankfurt am Main, Frankfurt/M. 1980, S. 25. Eintrag vom 11. April 1622, Frankfurt am Main: »Als Claudius D'Aquaviva, ein Medicus, abermals gebetten, Jhme Zuvergünstigen, daß er seine Artzneÿ offentlich, Jedoch ohne Music und Comoedien verkauffen möge. Soll man Jhme, doch daß er keine Thÿriac oder andere schädliche Sachen verkauffe, willfahren«, ebda.

15 Johann Baptista Montedor wurde zur Ostermesse 1625 zunächst abgeschlagen, »seine Medicamenta offentlich alhie $\mathrm{Zu}$ verkauffen vnd darbeÿ eine Music vnd Comoedien Zuhalten« und zwölf Tage später bewilligt, zit. n. H.R. Purschke, Puppenspiel, ebda., S. 26.

16 Tomaso Garzoni, La Piazza universale di tutte le professioni del mondo, hrsg. von Paolo Cherchi und Beatrice Collina, Bd. 2, Turin 1996, S. 1193. François Berthaud, La Ville de Paris en vers burlesques, Paris 1655, schreibt: »Vous, rendez-vous de charlatans, [...], Des chanteurs des chansons nouuelles [...], De ceux qui rendent des poulets«, zit. n. Jonathan Marks, »The Charlatans of the Pont-Neuf«, in: Theatre Research International 23 (1998), S. 133. Gaultier-Garguilles Chanson-Texte wurden 1631 gedruckt, siehe Chansons de Gaultier Garguille (wie Anm. 6).

17 Siehe Th. Coryate, Crudities (wie Anm. 11), S. 272ff. Thomas Platter d.J., Beschreibung der Reisen durch Frankreich, Spanien, England und die Niederlande, 1595-1600, hrsg. von Rut Keiser, Basel 1968 (Basler Chroniken, 9), Bd. 1, S. 306, schreibt: »Auch hatten sie gute spielleüt under ihnen selbs, konten die lauten, harpfen unndt violen gar lieblich zu ihrem eigenen gesang accomodieren, daß sich menneglich mußte dorab verwunderen.» Ottonelli spricht ebenfalls von »cantando o sonando« (wie Anm. 13), S. 456. Dasselbe siehe bei John Rastell, The third booke, declaring by examples out of Ancient councels, fathers, and later writers, that it is time to beware of $M$. Iewel, Antwerpen 1566, [S. 4].

18 T. Garzoni, Piazza universale (wie Anm. 16), S. 1195.

19 Siehe Anm. 17. 
»[...] etliche halten Tromen und Pfeiffen / etliche Trommeten / vnnd lassen bißweilen mit grossem Feldgeschrey zusamen blasen $[\ldots] \ll^{20}$

Die Abbildungen bestätigen und ergänzen diese Aussagen. Zwar ist es bei weitem nicht der Fall, dass, wie Nino Pirrotta schreibt, »each of the groups shows, generally, some musical instrument, such as a harp, a lute, a guitar, or a bowed instrument. $\ll^{21}$ Dennoch enthält das gesichtete Bildmaterial bereits 19 Abbildungen, die auf eine musikalische Tätigkeit der ciarlatani verweisen. Sie umfassen den zeitlichen Rahmen von der zweiten Hälfte des 16. bis zum frühen 17. Jahrhundert. Es ist davon auszugehen, dass die Abbildungen im Gegensatz zu den schriftlichen Quellen zwar weit weniger auf eine historisch-konkrete Situation verweisen, die Dargestellten stattdessen jedoch stilisiert zeigen, was wiederum die Charakteristika der Auftritte durch eine gewisse Typisierung unterstreicht. Von einer Dokumentationsfähigkeit der Bilder wird auch im Falle einer offensichtlich satirischen Betonung ausgegangen.

Den überwiegenden Teil der ältesten bisher bekannten Darstellungen musizierender ciarlatani bilden Zeichnungen in sogenannten Stammbüchern (Alba amicorum). Mit dieser Problematik hat sich insbesondere die britische Kunsthistorikerin Margret A. Katritzky befasst. Sie bietet in ihren Publikationen die bislang umfangreichste Sammlung derartiger Bildquellen. ${ }^{22}$ Der Aufbau der Bilder folgt einem mehr oder weniger feststehenden Schema (vgl. dazu auch die Abbildungen im Anhang). Auf einer kleinen Bank bzw. Bühne ist links zumeist ein Heilmittelverkäufer zu finden, rechts davon musizierende Komödianten. An Streichinstrumenten ${ }^{23}$ sind zumeist Fidel, Lira da braccio und Viola im Sinne des frühen Streichinstruments, ${ }^{24}$ darüber

20 Der 104. Diskurs ist überschrieben: »Von denen so allerhandt Schauspiel anstellen: sonderlich aber von Marck-Schreyern/Storgern und Theriackskrämern«, siehe Tomaso Garzoni, Piazza Vniversale: Das ist: Allgemeiner Schawplatz, oder Marckt vnd Zusammenkunfft aller Professionen, Künsten, Geschäfften, Händlen vnd Handtwercken, so in der gantzen Welt geübt werden: Deßgleichen Wann vnd von wem sie erfunden: Auch welcher massen dieselbige von Tag zu Tag zugenommen: Sampt außfiibrlicher Beschreibung alles dessen, so darzu gehörig: Beneben der darin vorfallenden Mängeln Verbesserung, vnd kurtze Annotation vber jeden Discurs insonderheit. Nicht allein allen Politicis, auch jedermänniglich, wes Standts sie seynd, sehr lustig zu lesen, Frankfurt/M. 1619, S. 579.

21 Nino Pirrotta, "Commedia dell'Arte and Opera«, in: N.P., Music and Culture in Italy from the Middle Ages to the Baroque. A Collection of Essays, Cambridge/Mass. 1984 (Studies in the history of music, 1), S. 344 .

22 Siehe dazu nachfolgende Veröffentlichungen: Margret A. Katritzky, »Italian Comedians in Renaissance Prints «, in: Print Quarterly 4 (1987), S. 236-254; dies., 》Scenery, setting and stages in late renaissance commedia dell'arte performances: some pictural evidence«, in: Scenery, set and staging in the Italian Renaissance: Studies in the Practice of Theatre, hrsg. von Christopher Cairns, Lewiston 1996, S. 209-288; dies., Was Commedia dell'arte (wie Anm. 12), S. 104-126.

23 Die Zuordnung der Streicher orientiert sich an Munrows Ausführungen über Streichinstrumente, siehe David Munrow, Musikinstrumente des Mittelalters und der Renaisance, Celle 1980, S. 130-139.

24 Siehe die beiden Titelkupfer der Tabarin-Werkausgaben um 1620 bzw. 1858 (vgl. Anm. 39) in: 
hinaus Laute, ${ }^{25} \mathrm{Harfe}^{26}$ und die Flöte ${ }^{27}$ nachweisbar. Auch im vorliegenden Fall stellt sich die Frage nach der Darstellung von Sängern bzw. Sängerinnen. Auf den Zeichnungen sind einzig und allein Art und Anzahl der Instrumente zu erkennen bzw. ob diese im Moment gespielt werden oder nicht. Eine Frage der Interpretation bleibt dagegen, ob die Bildzeugnisse singen.

Die Bildzeugnisse sind auch in der Hinsicht auf das musizierende Personal bemerkenswert: Die Instrumente werden in den wenigsten Fällen von den Heilmittelverkäufern selbst gespielt. Im überwiegenden Teil der Abbildungen tragen die Musizierenden Masken. Musikanten, die das Geschehen im wahrsten Sinne des Wortes >begleiten zu sehen, der die Bühne von Tabarin darstellt. ${ }^{28}$ Aus diesem Grund ist meines Erachtens zu vermuten, dass alle am Auftritt Beteiligten, so auch die Musiker, theatral aktiv gewesen sind.

Versucht man, das Bisherige zusammenzufassen, bleibt zu sagen, dass auf den Bühnen der ciarlatani mit einem relativ breiten Arsenal von Instrumenten Musik dargeboten wurde. Zudem ist eine solche Bühne in einer größeren Stadt keineswegs isoliert zu betrachten, sondern in den Zusammenhang des Marktplatzes einzubetten. Wie bereits erwähnt, schreibt Moryson: »all the Markett places of great Cittyes are full of Montebanks, or Ciarlatanes «, ${ }^{29}$ und Thomas Coryate spricht im Jahre 1611 von fünf bzw. sechs gleichzeitig aufgebauten Bühnen, ${ }^{30}$ ebenso wie Ottonelli im Jahre 1652. ${ }^{31}$

Demnach war Musik, ob sie nun auf der ciarlatani-Bühne selbst oder in deren Umfeld gespielt wurde, Teil des Auftrittes bzw. im Umfeld beteiligt. Anhand der Quellen, insbesondere an Platter und Coryate, wird deutlich, dass diese keinesfalls nur zur Anlockung der Kunden diente, sondern den gesamten Auftritt strukturierte und beispielsweise auch während des Verkaufs der Medikamente und danach eingesetzt wurde.

Commedia dell'Arte. Eine Bildgeschichte der Kunst des Spektakels, hrsg. von David Esrig, Nördlingen 1985, S. 53.

25 Siehe Abb. 1 und 2. Siehe auch Giacomo Franco, Komödianten und Scharlatane auf dem Markusplatz in Venedig (1610), in: N. Pirrotta, Commedia dell'Arte (wie Anm. 21), S. 344.

26 Ebda.

27 Anonym, Gaukler und Musikanten in Venedig, Gouache mit Goldhöhung, 20,5 x 13,7 cm, aus einem Stammbuch um 1600, Staatsbibliothek Bamberg (D-BAs, I. Qc. 75). Das Bild liegt ebenfalls gedruckt vor, als Beilage zu TIBLA - Magazin für Holzbläser 4 (1997).

28 Commedia dell'Arte (wie Anm. 24), S. 53.

29 Wie Anm. 2.

30 Wie Anm. 11.

31 Wie Anm. 13. 


\section{Musikalisches Material}

Nun stellt sich selbstverständlich die Frage, was musiziert wurde. Heinrich Besseler behauptet im Kapitel »Von Lasso zu Giovanni Gabrieli« seiner Schrift Die Musik des Mittelalters und der Renaissance, ${ }^{32}$ dass die italienischen ciarlatani Villanellen gesungen haben. Dieser Äußerung muss jedoch hinzugefuigt werden, dass sie in der genannten Eindeutigkeit bisher nicht durch Quellen zu belegen ist. Es handelt sich vielmehr um einen Analogieschluss.

Es ist bekannt, dass comici dell'arte commedie alla villanesca gespielt ${ }^{33}$ oder, wie Orlando di Lasso im Jahre 1568 als Pantalone, Villanellen gesungen haben. Angesichts der engen Verbindung zwischen ciarlatani und commedia dell'arteerinnert sei allein daran, dass man den berühmten commedia-dell'arte-Spieler Tristano Martinelli als Oberhaupt der ciarlatani bezeichnete ${ }^{34}$ - erscheint die Verwendung von Villanellen demnach einigermaßen plausibel. Bianca Maria Galanti geht im Übrigen davon aus, dass die von ihr untersuchten populären Villanellen-Drucke von ciarlatani verkauft worden sind. ${ }^{35}$

\section{Die Art und Weise des Musizierens}

Die Quellen beantworten statt der Frage, was gespielt wurde, eher die Frage nach der Art und Weise, wie gespielt wurde. An erster Stelle verdient hier Garzonis Aussage in der Piazza universale aus dem Jahre 1585 Aufmerksamkeit, die ciarlatani

32 »Als strophische Dialektdichtung von teilweise höchst drastischer Art, beheimatet in den Volkskomödien neapolitanischer Possenreißer und ,Ciarlatani<, vertrat sie in ihrer aufreizend-neuartigen musikalischen Einkleidung die Gegenwelt zum niederländischen Kontrapunkt.« Heinrich Besseler, Die Musik des Mittelalters und der Renaissance, Potsdam 1931 (Handbuch der Musikwissenschaft), S. 300.

$33 \mathrm{Vgl}$. Alfred Einstein, The Italian Madrigal, Princeton 1949, Bd. 1, S. 340 und 344.

34 Tristano Martinelli »occupava a Mantova la carica di s superiores di tutti i scomici mercenari, zaratani, cantinbanco, bagattiglieri, postiggiatori, et che mettono banchi per vender ogli, balotte, saponeti, historie et cose simili [...] sì che alcuno di loro, o solo o accompagnato, sia di che paese esser si voglia, non habbia ardire de recitare comedie o cantar in banco vendendo ballotti, far bagatelle, posteggiare in terra o metter banco senza licenza di detto Martinelli in scrito, né d'indi partirsi senza la medesima licenza, sotto pena d'essere spogliati di ciò ch'avranno così comune come proprio«, zit. n. S. Ferrone, Attori (wie Anm. 10), S. 11.

35 Bianca Maria Galanti, Le villanelle alla napolitana, Florenz 1954 (Biblioteca dell'Archivum romanicum, 1.39), S. XVII-XVIII. Dass Quacksalber Liebeslieder gedruckt haben, wird ebenfalls berichtet, vgl. Claude-Stéphen Le Paulmier, Docteur Le Paulmier. L'Onviétan. Histoire d'une famille de charlatans du Pont-Neuf, Paris 1893, S. 10ff. Coryate hatte im Übrigen berichtet, die ciarlatani in Venedig hätten gedruckte Liebeslieder verkauft (wie Anm. 11), S. 274. 
hätten »all'improviso gesungen. ${ }^{36}$ Das »cantare all'improviso« gibt die deutsche Ausgabe aus dem Jahre 1619 im übrigen mit »lieblichem Gesang « wieder. ${ }^{37}$

Thomas Platter der Jüngere bemerkt hinsichtlich der aus sieben Komödianten bestehenden Truppe des Ian Braghetta:

Auch hatten sie gute spielleüt under ihnen selbs, konten die lauten, harpfen unndt violen gar lieblich zu ihrem eigenen gesang accomodieren, daß sich menneglich mußte dorab verwunderen. ${ }^{38}$

Eine weitere Quelle findet sich in den sogenannten Euvres de Tabarin. In den darin abgedruckten Tromperies des charlatans von Thomas Sonnet de Courval ist über Hieronymo Ferranti, der vermutlich Anfang des 17. Jahrhunderts aus Rom nach Paris kam, zu lesen:

il avoit quatre excellens joueurs de violon qui avoient séance aux quatre coings de son theatre, lesquels faisoient merveilles, assistez d'un insigne bouffon ou plaisant de l'hostel de Bourgogne nommé Galinette la Galina.

Weiter unten wird nochmals »la douce harmonie et harmonieuse douceur des instrumens" hervorgehoben. Thomas Coryate berichtet von einem »mountebank«, der berühmt dafür war, ein außergewöhnlich guter Sänger von »extemporall songs « zu sein. ${ }^{40}$ Von Claudius de Aquaviva schließlich heißt es im Jahre 1622, er habe »den Käufern [...] eine liebliche Musika« gespielt. ${ }^{41}$

Selbstverständlich ist es nicht unproblematisch, aus diesen Beschreibungen Schlüsse zu ziehen. Es wäre voreilig, das heutige Verständnis von lieblicher, wohlklingender Musik auf diese Quellen zu übertragen. Festzuhalten bleibt jedoch, dass die Zeitgenossen die Musik auf den Bühnen der ciarlatani als wohlklingend empfanden, keineswegs als störend. Das Instrumentarium der Ab- bildungen lässt durchaus Rückschlüsse auf eine wohlklingende Musik zu. Keine einzige bildet Blas- oder Schlaginstrumente ab.

Von anderer Qualität spricht beispielsweise Coryate, als er von dem »fellow erzählt, der bekannt war für »a pretty kinde of musicke that he made with two bones betwixt his fingers«. ${ }^{42}$ Garzoni berichtet: »Fornita questa istoria, Gradella

36 T. Garzoni, Piazza universale (wie Anm. 16), S. 1193.

37 T. Garzoni, Allgemeiner Schawplatz (wie Anm. 20), S. 578.

38 Th. Platter, Beschreibung (wie Anm. 17), S. 306. Weiter unten, den Quacksalberauftritt selbst betreffend, schreibt Platter: »Alsdann spilen sie ettwas liebliches mitt einanderen auf ihren instrumenten undt singen lieblich darunder «, ebda.

39 Thomas Sonnet de Courval, "Les tromperies des charlatans« [1629], in: EEvvres complètes de Tabarin avec les rencontres, fantaisies et coq-à-l'âne facétieux du Baron de Gratelard et divers opuscules, hrsg. von Gustave Aventin, Paris 1858 (Bibliothèque Elzévirienne, 61), Bd. 2, S. 213.

40 Th. Coryate, Crudities (wie Anm. 11), S. 274.

41 Siehe die Akteneinträge vom 16. November 1621 und vom April 1622, zit. in Anm. 14.

42 Th. Coryate, Crudities (wie Anm. 11), S. $274 \mathrm{f}$. 
fa una squaquarata di voce e di canto molto sonora, overo finge l'orbo col cagnuolo in mano in luogo di tiorba. ${ }^{43}$ All dies scheint der Unterhaltung keinen Abbruch getan zu haben. Regelrechte Beschwerden insbesondere über die musikalische Seite der ciarlatani setzen nach meiner Kenntnis erst im späten 17. bzw. 18. Jahrhundert ein. ${ }^{44}$

\section{Funktion der Musik beim Auftritt der ciarlatani}

In das Zentrum der Aufmerksamkeit rückt nunmehr die Frage, welche Funktion die Musik beim Auftritt von ciarlatani erfüllte. In den Quellen finden sich dazu verschiedene Aussagen. Kritische Stimmen warfen den ciarlatani seit dem späten 17. Jahrhundert z.B. vor, sie hätten sich der Musiker und Komödianten immer nur dann bedient, wenn ihre ärztliche Scharlatanerie nicht genügend Geld einbrachte. ${ }^{45}$ Während diese Einwände nur sporadisch auftauchen bzw. den Zorn über nicht zu verhehlenden Erfolg erkennen lassen, war eine andere Begründung weitaus folgenreicher. Von mehreren Reisenden, ${ }^{46}$ so unter anderem von dem Franzosen Sonnet de Courval, ${ }^{47}$ wird das Argument ins Feld geführt, die ciarlatani spielten Musik zur Anlockung ihrer Kunden.

Die Geschichtsschreibung wie auch die neuere Forschung hat sich dieser Begründung angenommen, ${ }^{48}$ und zwar so beharrlich, als ob ihr etwas Pejoratives anhaften würde. Ebenso wurde mit dem bereits erwähnten Moment der Ablenkung verfahren, das ohne Zweifel auf zwei Ebenen funktioniert haben dürfte, nämlich in Bezug auf den Patienten und in Bezug auf das Publikum. Allerdings

43 T. Garzoni, Piazza universale (wie Anm. 16), S. 1194. Wenig später berichtet er von Settecervelli, der sprende occasione di far circolo, e con la cappa distesa per terra, con la cagnola appresso, con la bacchetta in mano, la fa cantare ut, re, mi fa, sol, la, le fa far tombole per galanteria«, ebda., S. 1196.

44 Siche Maren Goltz, „Zur musikalischen Seite von Quacksalbern«, in: Theater und Heilkunst. Studien zu Theater und Anthropologie, hrsg. von Gerda Baumbach, Weimar 2001 [im Druck].

45 Th. Sonnet de Courval, Les tromperies (wie Anm. 39), S. 137. Johann Valentin Neiner, Laecherlich =jedoch vernuenffiger, bescheidener und curioser Narren $=$ Calender, Auf das Jahr 1712 , Köln 1712, [fol. 46].

46 Siehe Garzonis 104. Kapitel »Von denen / so allerhandt Schawspiel anstellen: sonderlich aber von Marck=Schreyern / Storgern und Theriackskrämern (wie Anm. 20), S. 575-580. Ottonelli beschreibt dies als »ad allettar il popolo« (wie Anm. 13), S. 456.

47 Th. Sonnet de Courval, Les tromperies (wie Anm. 39), S. 213.

48 Fournier spricht vom »amassant la poulace«, siehe Chansons de Gaultier Garguille (wie Anm. 6), S. liv. Jacob spricht vom »Anlocken seiner Kunden«, siehe Martin Jacob, Kölner Theater im XVIII. Jahrhundert bis zum Ende der reichsstädtischen Zeit (1700-1794), Emsdetten 1938 (Die Schaubühne, 21), S. 3. Siehe auch Arthur Kopp, Eisenbart im Leben und im Liede, Berlin 1900 (Beiträge zur Kulturgeschichte, 3), S. 4f. Siehe auch M.A. Katritzky, Was Commedia dell'arte (wie Anm. 12), S. 119, und J. Marks, The Charlatans (wie Anm. 16), S. 133. 
bleibt generell zu fragen, inwieweit die »liebliche Musica« ein probates Mittel darstellt, Schmerzensschreie zu übertönen.

Durch die Reduktion auf Anlockung bzw. Ablenkung wird meines Erachtens ein ganz wesentlicher Punkt überdeckt. Anzunehmen und mehrfach zu belegen ist, dass sich das Publikum des Auftrittes gut unterhalten fühlte. Und daran hat die Musik einen wesentlichen Anteil gehabt.

\section{Das Publikum und die Wirkung des Auftritts}

Über das Publikum der ciarlatani lässt sich grundsätzlich sagen, dass es zunächst einmal relativ wählerisch sein konnte. Statt einen bereits gezahlten Eintritt aussitzen zu müssen, stand es vor der Bühne und hatte im besten Fall, wenn es sich um eine größere Stadt handelte, sogar die Möglichkeit, sich binnen kürzester Zeit für einen zweiten und dritten Auftritt von ciarlatani zu entscheiden.

Die tatsächliche Reaktion des Publikums bleibt bislang jedoch weitgehend im Dunkeln. Die Quellen geben weder Auskunft, ob die Umstehenden etwa zum Singen oder zum Tanzen animiert wurden, noch, in welcher Befindlichkeit sie nach Hause gegangen sind. Ersichtlich ist aus den Beschreibungen im höchsten Fall, dass die Leute von den ciarlatani gut unterhalten wurden und dass sie gelacht haben. Ottonelli beschreibt dies als »festa, con riso e con sollazzo ${ }_{,}^{49}$ und bei Platter ist zu lesen, dass "yedreman genug ze lachen hatte unndt gar gern zu sahe . $^{50}$ Coryate berichtet, dass ihm die ciarlatani »oftmals unendliches Vergnügen bereiteten « und "in der ganzen Menge große Heiterkeit « hervorriefen. ${ }^{51}$ Wäre dies nicht der Fall gewesen, hätte sich das zahlreiche Publikum gewiss nicht, wie in den Quellen geschildert, bis zu mehreren Stunden vor den Bühnen der ciarlatani aufgehalten. ${ }^{52}$

Claudius de Aquaviva ist meines Wissens der einzige ciarlatano, der diese Funktion seines Musizierens reflektiert, indem er angibt, seine »liebliche Musica«

49 G.D. Ottonelli, Della christiana moderatione (wie Anm. 13), S. 456.

50 Th. Platter, Beschreibung (wie Anm. 17), S. $305 \mathrm{f}$.

51 Th. Coryate, Crudities (wic Anm. 11), S. 272.

52 Garzoni betont: "I ceretani adunque [...] fra la vilissima plebe s'hanno acquistato ormai credito tale che molto maggior concorso con piú lieto applauso si fa loro ch'agli eccellenti oratori del verbo divino e agli onorati catedranti delle scienze e arti ingenue, di picciola corona rispetto a loro circondati intorno« (wie Anm. 16), S. 1188. Weiterhin ist zu lesen, dass sie »ogni sera dalle vintidue fino alle vintiquattro ore di giorno auftraten, ebda., S. 1192. Bei Platter ist zu lesen, er hätte: »ettwan ein par stundt auf demselbigen tisch ein lustige comoedien gespilet unndt gesehen, daß eine grosse menge volcks beyeinander wahre, von 100 biß in die 500 oder auch 1000 personen « (wie Anm. 17), S. 306. Sonnet de Courval spricht von »la foulle« (wie Anm. 39), S. 137. Ottonelli schreibt, dass die Leute etwa zwei Stunden stehen blieben (wie Anm. 13), S. 456. 
und »Italienische comedia« »den Käufern zur Danksagung undt zur recorirung des Gemüths « zu spielen. ${ }^{53}$ Musik wird von Aquaviva, in Verbindung mit dem Comödien-Spiel, quasi als Heilmittel begriffen.

Argumentieren wir also mit dem Konstrukt eines idealen Auftritts von ciarlatani. Das Mobilisieren von Kräften, die durch Freude, Wohlbefinden und Lachen ausgelöst werden, ist nicht zu trennen vom Fernhalten von Krankheit. ${ }^{54}$ Bei der ständigen Wiederholung der Anlockungs- und Ablenkungsthese wird dies unterschlagen.

Der hohe Entlastungswert von Musik ist seit Menschengedenken bekannt. Musik hat unmittelbare Wirkung auf das der bewussten Kontrolle entzogene vegetative Nervensystem, beeinflusst Herztätigkeit, Atemfrequenz, Blutdruck etc. ${ }^{55}$ Es liegen, wie Werner Friedrich Kümmels Studie über den Zusammenhang von Musik und Medizin beweist, zahllose Beispiele furr das Heilen mittels Musik vor. Formen gezielt heilenden musikalischen Theaters sind aus dem Mittelalter bekannt. ${ }^{56}$ Belege dafür liegen aber auch aus späterer Zeit vor. Im Jahre 1569 etwa verordnete der französische Wundarzt und Chirurg Ambroise Paré seinem Patienten »Violen, Violinen und einen Possenreißer«, und zwar mit dem erklärten Ziel, »ihn aufzuheitern «. ${ }^{57}$

Dass ähnliche Quellen eher selten nachgewiesen sind, mag neben dem bisher anders gelagerten Forschungsinteresse unter anderem auch daran liegen, dass die Einheit Musiker/Spieler/Heiler selbstverständlich war und nicht, wie im vorliegenden Fall, der besonderen Erwähnung bedurfte. Der bereits zitierte Sonnet de Courval berichtet 1619 ausdrücklich, Galinette la Galina habe Ferrantis vier Violinisten assistiert. ${ }^{58}$

Um es mit Platters Worten zu sagen:

Viel dergleichen unndt andere wunderbahre spiel unndt däntz tragen sich da oftermahlen zu, welche nitt alle kennen in die federen gefaßet werden. ${ }^{\text {so }}$

53 Siehe die Akteneinträge vom 16. November 1621 und vom April 1622, zit. in Anm. 14.

54 Stefanie Hüttinger, Die Kunst des Lachens - das Lachen der Kunst. Ein Stottern des Körpers, Frankfurt/M. 1996 (Europäische Hochschulschriften, 30.65).

55 Kurt Blaukopf, Musik im Wandel der Gesellschaft. Grundziuge der Musiksoziologie, ${ }^{2}$ Darmstadt 1996, S. 166. Er schließt daran an: »Hieraus ist die magische und extatische Wirkung von Musik zu erklären.«

56 Werner Friedrich Kümmel, Musik und Medizin. Ihre Wechselbeziehungen in Theorie und Praxis von 800 bis 1800, Freiburg 1977 (Freiburger Beiträge zur Wissenschafts- und Universitätsgeschichte, 2). Siehe auch Walter Salmen, Der Spielmann im Mittelalter, Innsbruck 1983 (Innsbrucker Beiträge zur Musikwissenschaft, 8), S. 83.

57 W.F. Kümmel, Musik, ebda., S. 385.

58 Th. Sonnet de Courval, Les tromperies (wie Anm. 39), S. 213.

59 Th. Platter, Beschreibung (wie Anm. 17), S. 308. 
Generell ist Folgendes festzuhalten: Salmens Feststellung, die »durch musikantisches Spiel am leichtesten erzielbare Wirkung " sei Freude und Kurzweil ${ }^{60}$ ist meines Erachtens über die Grenzen des von ihm untersuchten Zeitraumes hinaus gültig. Die zugrunde liegende Strategie zielt auf eine Wirkung sjenseits des Intellekts, hinter dem analytischen Verstand ${ }^{61}$ ab: »Nicht an anspruchsvolle Kunstmusik war gedacht«, zumindest nicht in erster Linie, sondern »an das ganze große Gebiet des [...] Gesangs und Instrumentalspiels, das nicht zur Ars musica gehörte «, vor allem an »leicht eingängige Stücke, Lieder und Tänze, mit denen die Spielleute und >Bierfiedler< aufwarteten und die auch musikalische Laien vortragen konnten. Damit wurde einer natürlichen Veranlagung des Menschen Rechnung getragen, die von musikalischer Bildung unabhängig ist. $\ll^{62}$

Gestuitzt wird die These von der heilenden Funktion von Musik beim Auftritt von ciarlatani zusätzlich durch Pirrottas Hinweis: "In this case, at the time of its performance everyone knew the music performed and the ways and means of its execution $\kappa^{63}$ Von dieser Überlegung ist es nur ein kurzer Weg zu Carl Somborn, der das stete Anhören solcher beliebter Melodien, denen alle möglichen Texte unterlegt wurden, mit körperlichem Wohlbehagen gleichsetzt. ${ }^{64}$

Im Idealfall diente die Musik also keineswegs nur der Anlockung, sondern trug ganz wesentlich zum Wohlbefinden und zur Gesundheit des Publikums bei. In Garzonis Schilderung des ciarlatano heißt es:

che da un canto della piazza tu vedi il nostro galante Fortunato insieme con Fritata cacciar carotte, e trattener la brigata ogni sera dalle vintidue fino alle vintiquattro ore di giorno, finger novelle, trovare istorie, formar dialoghi, far caleselle, cantare all'improviso, corrucciarsi insieme, far la pace, morir dalle risa, alterarsi di nuovo, urtarsi in sul banco, far questione insieme, e finalmenta buttar fuora i bussoli, e venire al quanquam delle gazette che vogliono carpire con queste loro gentilissime e garbatissime chiacchiere. ${ }^{65}$

60 Salmen schreibt: mfröüd und kurzwil zemachen««, siehe W. Salmen, Der Spielmann (wie Anm. 56), S. 83. "Diese Elementareigenschaft, an Stelle von strûren< belebende sfröude <u verbreiten, hatte wohl auch Jacobus von Lüttich (Speculum musicae I,5) im Sinn, wenn er drastisch schrieb: ,Qui per musicam non afficitur, bestialis vel plus quam bestia fore videtur. sIch ergötze die Menschen< war bereits ein Grundgebot des homerischen Sängers, im Mittelalter kamen >Budours into the haulle tay brynge that gayly with thaire gle gan synger (Rowland and Ottuel, 34) «s ebda.

61 Ebda., S. 82.

62 W.F. Kümmel, Musik (wie Anm. 56), S. 387.

63 Über die Bekanntheit der Musik der comici dell'arte sagt Pirrotta: "In this case, at the time of its performance everyone knew the music performed and the ways and means of its execution, but time has swallowed and buried this direct knowledge and has left us only scattered and secondor third-hand documents« (wie Anm. 21), S. 344.

64 »Aber freilich kann durch das stete Anhören solcher >beliebter < Melodien, denen alle möglichen und unmöglichen Texte untergelegt werden, im Grunde lediglich das körperliche Wohlbehagen gefördert werdenk, siehe Carl Somborn, Das Venezianische Volkshied, die Villotta, Heidelberg 1901, S. 47.

65 T. Garzoni, Piazza universale (wie Anm. 16) S. 1192f. In der deutschen Ausgabe ist zu lesen: 
Garzoni nennt in diesen Zeilen sehr verschiedene Bestandteile des Auftritts. Bereits anhand des Zitats wird deutlich, dass es kurzsichtig wäre, sich auf eine Sonderstellung der Musik einzulassen. ${ }^{66}$ Denn es geht nicht um eine autonome, sondern in jedem Fall um eine zweckgerichtete Musik. Nicht Gattungsfragen stehen im Mittelpunkt des Interesses, auch nicht in erster Linie der Einsatz von lieblicher Musik und Lärm, sondern der Umgang mit Musik im gesamten Ereignis und ihr Verhältnis zu anderen Bestandteilen. Entscheidend ist, wie sich die Musik in den Kontext des Auftrittes einordnet: Wie ist die Strategie des Auftritts zu formulieren? Welche Rolle spielte das Gegeneinandersetzen von Spieltechniken? Welche Wirkungen wurden mit der Technik dieses immer neuen Komponierens erzeugt?

So weit wie möglich fassen kann man die Strategie des gesamten Auftritts meiner Ansicht nach, indem man sie als ein Verfahren des Zusammensetzens, des Vermischens, des $" Z$ Zusammenfummelns $\ll^{67}$ und des Springens bestimmt, kurz des Wechselspiels zwischen den einzelnen am Auftritt beteiligten Bestandteilen des Auftritts, wie sie z.B. von Garzoni benannt werden. Musik ist dabei einer von zahlreichen Komponenten, die miteinander interagieren. Dass die Grenzen zwischen ihnen nicht starr verlaufen, bedarf keiner Erwähnung, macht die Kompliziertheit der Fragestellung jedoch erneut deutlich. Dass sich Musik für dieses Verfahren der Parodie in besonderer Weise eignet, liegt auf der Hand.

Gerda Baumbach spricht in Zusammenhang mit dem »Gil-Vicente-Salat«, der einem liebeskranken Priester dargebracht wird - einem Quodlibet und »Durcheinander aus bekannten Volksliedern« in der 1524 uraufgeführten Farga dos Físicos des Gil Vicente -, von »einem vermutbaren direkten Zusammenhang « des Quodlibets als Ragout und Salat »mit einer leiblich wohltätigen, ssüßen< und heilenden Wirkung ${ }^{68}{ }^{6}$

»bald mit einer newen Zeitung / baldt mit einer Historien / bald mit einem Dialogo, bald mit einem lieblichen Gesang / bald hadert er mit seinem Knecht / bald versöhnet er sich wider mit ihm / baldt lachet er / daß ihm die Augen uberlauffen / [...] alsdenn bringt er seine Büchslein herfür / und kompft auff sien quamquam von den Hellern / die er gerne hette / unnd fängt an seine herzliche Wahren zu loben / unnd treibet solches so lang / biß er etliche uberredet / daß sie ihm was abkauffen.« (wie Anm. 20), S. 578.

66 Zur Rolle der Musik bei Garzoni siehe im Übrigen Massimo Privitera, »Hospidale de'pazzi e Comedia harmonica - Musica e letteratura democritea in Emilia-Romagna alla fine del ' 500 «, in: Tomaso Garzoni. Uno zingaro in convento. Celebrazioni garzoniane, IV centenario (1589-1989), Ravenna-Bagnacavallo 1989-1990, Ravenna 1990 (Il portico, 93), S. 81-108.

67 Christian Kaden, Des Lebens wilder Kreis. Musik im Zivilisationsprozeß, Kassel 1993, S. 53.

68 Gerda Baumbach, Seiltänzer und Betrüger? Parodie und kein Ende. Ein Beitrag zu Geschichte und Theorie von Theater, Tübingen 1995 (Mainzer Forschungen zu Drama und Theater, 13), S. 9. Bereits Holl sprach in Zusammenhang mit dem Erfolg der englischen Komödianten in Deutschland von ihrem aus »Singewerk, Spielwerk, Feuerwerk « zusammengebrauten "Ragout" und zitiert Johannes Rhenanus, der in der Vorrede zu seiner Komödie Speculum aistheticum 
Interessanterweise ist eine musikalische Quelle überliefert, die als Argument fuir die hier aufgestellte Theorie des Heilens dienen kann. Sie untermauert neben der Verbindung zur commedia dell'arte den Gedanken von der Strategie. Es handelt sich um Giovanni Croces Triaca musicale ${ }^{69}$ aus dem Jahre 1596. Die Capricci genannten Ingredienzien des wundertätigen Balsams sind ein Auftritt von Pantalone, der Wettstreit zwischen einem Kuckuck und einer Nachtigall, ein Kinderlied, eine mascherata paduanischer Bauern, die Versteigerung einer Sklavin und ein Würfelspiel. Alfred Einsteins Kommentar zum Musikalischen Theriak lautet: "The music is as simple as possible: this is entertainment, not art. ${ }^{70}$

$\mathrm{Zu}$ entscheiden bleibt freilich, inwieweit sich über das Konstatieren einer solchen Mischtechnik hinaus bestimmte Fragen der wissenschaftlichen Aufarbeitung entziehen, wie etwa jene nach dem Anteil einzelner Bestandteile am spielerischen Austausch auf allen Ebenen des Ereignisses. Jegliche Festlegung würde Flexibilität, Variabilität und Wandelbarkeit, das ,So-und-auch-anders ‘ gefährden.

\section{Zusammenfassung und Ausblick}

Trotz des dringenden Bedarfs, die einzelnen Ergebnisse auf eine breitere Quellenbasis zu stellen und etwa in zeitlicher und regionaler Hinsicht stärker zu differenzieren, kann bisher gesagt werden, dass die herangezogenen Quellen die These vom strukturbildenden Anteil der Musik an der Wirkungsstrategie der ciarlatani bestätigen.

Entgegen den mir aus der Zeit der Renaissance bekannten Quellen gibt es in späteren Jahren durchaus kritische Stimmen zur musikalischen Tätigkeit auf den Bühnen der ciarlatani. In seiner polemischen Schrift Bellum Musicum vom Ende des 17. Jahrhunderts lässt der Weißenfelser Kapellmeister Johann Beer die aus Griechenland angereiste Compositio einen Klagebrief an ihre Tochter Harmonia schreiben, worin sie die Leiden schildert, die ihr in Deutschland zugefügt werden. Auf dem Höhepunkt angelangt, empört Compositio sich schließlich, dass sie »ja gar zu denen Aertzten auf öffentlichen Marckbuden geführet « wird. ${ }^{71}$ Soviel zur unwürdigen Behandlung der hohen Dame Compositio.

(1613) schreibt: „So wird der beste Trank gebraut, Der alle Welt erquickt und auferbaut «, siehe Karl Holl, Geschichte des deutschen Lustspiels, Leipzig 1923, S. $78 \mathrm{f}$.

69 Giovanni Croce, Triaca musicale (1596). Nella quale vi sono diversi capricci a 4, 5, 6, 7 voci miste, hrsg. von Achille Schinelli, Rom 1942 (Capolavori polifonici del secolo XVI, 3).

70 A. Einstein, The Italian Madrigal (wie Anm. 33), S. 801.

71 Johann Beer, Bellum Musicum oder Musicalischer Krieg, In welchem umbständlich erzehlet wird, wie die Konigin Compositio nebst ihrer Tochter Harmonia mit denen Hümpern und Stümpern zerfallen, und nach beyderseits ergriffenen Waffen zwey blutige Haupt-Treffen sambt der Belagerung der Vestung Systema unfern der Invention-See vorgegangen, auch wie solcher Krieg endlich gestillet, und 
Für das Phänomen des ciarlatano gilt exemplarisch, was Christian Kaden für die letzten beiden Jahrtausende europäischer Kulturgeschichte andeutet: »Interaktive Sozialstrukturen [...] werden ihr zunehmend, ja in katastrophischem Umfange problematisch. $\aleph^{72} \operatorname{Im} 18$. Jahrhundert löst sich diese Verbindung von Ärzten, Komödianten und Musikern: Alle Beteiligten spezialisieren und etablieren sich. In sehr vereinfachter Form bedeutet dies für das Theater: Man verzichtet zunehmend zugunsten regelmäßig gebauter Stücke auf die Mischtechniken. Der direkte Kontakt von Publikum und Spielern, etwa durch den Tausch von Heilmitteln gegen Geld, wird auf lange Sicht unterbrochen.

Hier offenbart sich ein weiteres, hochinteressantes Forschungsgebiet, dessen Untersuchung wichtige Aufschlüsse verspricht: Über welche Zeiträume hat man sich derartiger Mischtechniken bedient? Und: Wie lange hält sich in den festen Theaterhäusern von Seiten der Spieler der konkrete Anspruch der Rekreation bzw. Regeneration des Publikums?

Es liegt nahe, dass entlang der von Kurt Blaukopf diagnostizierten »Mutation des musikalischen Verhaltens $\ll^{73}$ und der zunehmenden Etablierung des Musikers ${ }^{74}$ empfindliche Veränderungen, um nicht zu sagen Abtrennungsvorgänge vonstatten gegangen sind. Dass die sich vollziehenden Veränderungen Spuren im Verhalten des Publikums hinterlassen haben, ist unbestritten. Blaukopf hat dies mit den folgenden Worten nachdrücklich zusammengefasst: $»$ Die Pose, die der musikalisch gebildete europäisch-abendländische Musikhörer bei der Darbietung eines musikalischen Kunstwerks einzunehmen sucht, läßt die[se] ursprüngliche Einheit von Musik, Atmung, Herzschlag und Bewegung leicht vergessen. $\ll^{75}$

der Friede mit gewissen Grund-Reguln befestiget worden, Denen von Musicalischer Hostilität allenthalben infestierten Frontir-Palätzen zum besten, und diesen, welche von der Music einzigen Aestim machen, nicht ohne dem daraus entspringenden Nutzen zu Liebe auf das kürtzeste entworffen, und mit einer Landt-Carte des Cymbalischen Reichs versehen, 1701, S. 3.

72 Ch. Kaden, Des Lebens wilder Kreis (wie Anm. 67), S. 61.

73 K. Blaukopf, Musik (wie Anm. 55), S. 167.

74 Es ist bezeichnend, dass der neuzeitliche Terminus Musiker eine Veränderung anzeigt. Grimm versieht die Erklärung mit dem Kommentar: smit edelm beiklange«. Jacob und Wilhelm Grimm, Deutsches Wörterbuch, Leipzig 1893, Reprint Gütersloh 1991, Bd. 12, Sp. 2742. Durch die Spezialisierung bzw. das Auseinanderbrechen der Gemeinschaft Musiker/Possenreißer geht die Wirksamkeit zunehmend verloren. Aus dem Verständnis von Musik wird eine wesentliche Ebene ausgeblendet. Musizieren gehört nur noch in sehr beschränktem Maße zur wie auch immer gearteten sreinen< Theaterkunst im weitesten Sinne. Zur sEmanzipation< des Musikers siehe Walter Salmen, Beruf: Musiker. Verachtet - vergöttert - vermarktet. Eine Sozialgeschichte in Bildern, Kassel 1997, passim. Hermann Schwedes, Musikanten und Comödianten - eines ist Pack wie das andere. Die Lebensformen der Theaterleute und das Problem ibrer bürgerlichen Akzeptanz, Bonn 1993 (Orpheus-Schriftenreihe zu Grundfragen der Musik, 65), passim.

75 K. Blaukopf, Musik (wie Anm. 55), S. 167. 


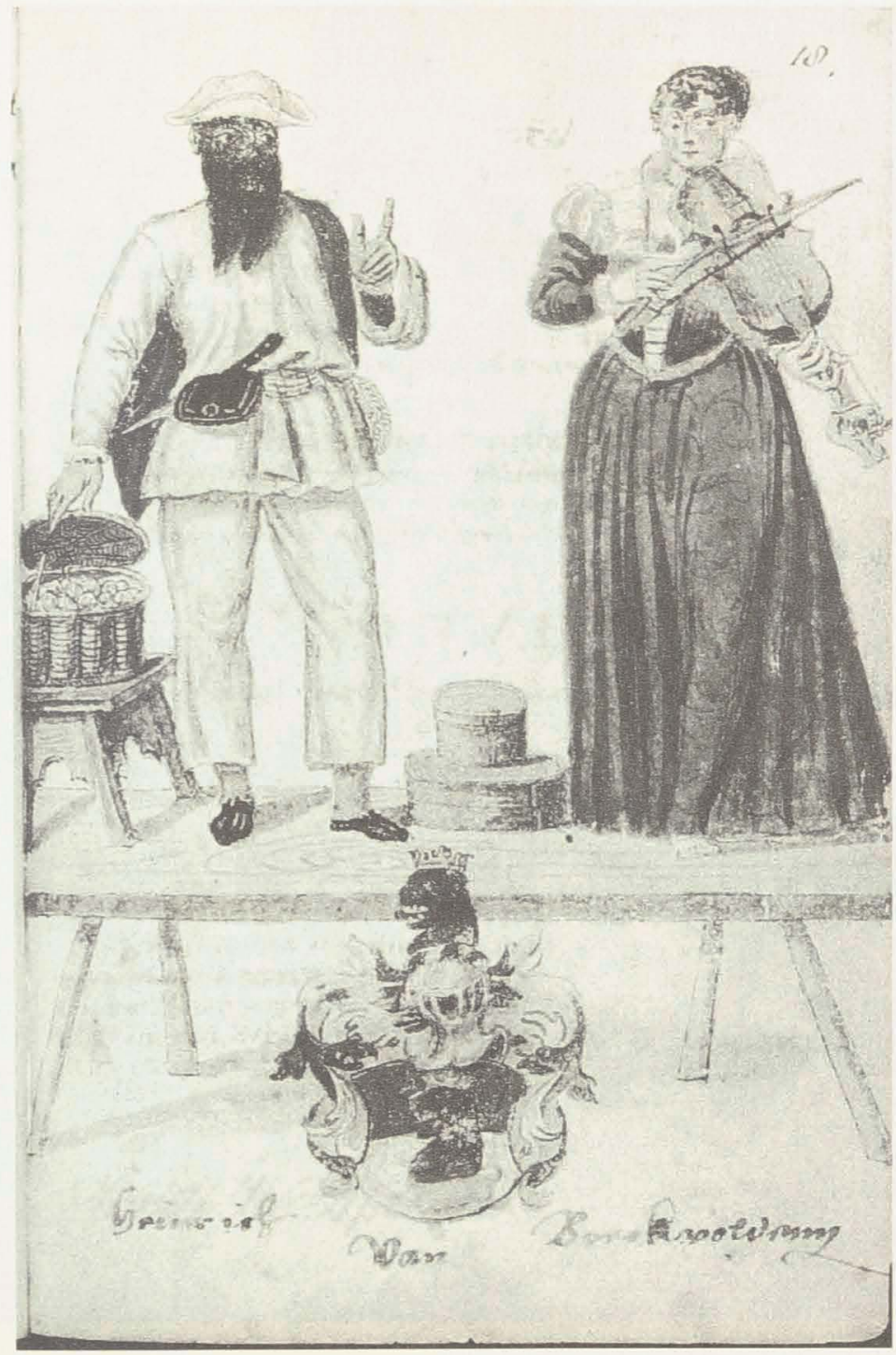

Abbildung 1: Album amicorum von Sigmundus Ortelius, 1573-1579, London, British Library (GB-Lbl, Ms. Eg.1191), fol. 18', siehe auch M.A. Katritzky, Was Commedia dell'arte (wie Anm. 12), pl. 2.

Die Maske auf der linken Seite hält den Deckel eines Korbes offen, dessen Inhalt nicht genau auszumachen ist. Die Frau auf der rechten Seite musiziert mit einem konkaven Bogen auf einer Lira da braccio mit einer tiefen Saite neben dem Griffbrett. Der Korpus der Lira da braccio weist zwei leicht f-förmige Schallöcher auf. Die Saitenanzahl ist nicht zu bestimmen. Zu diesem weitgehend auf Italien beschränkten Instrument, das auf Bildzeugnissen oft nur schwer von der Renaissance-Fidel zu unterscheiden ist, siehe D. Munrow, Musikinstrumente (wie Anm. 23), S. 130-139. 


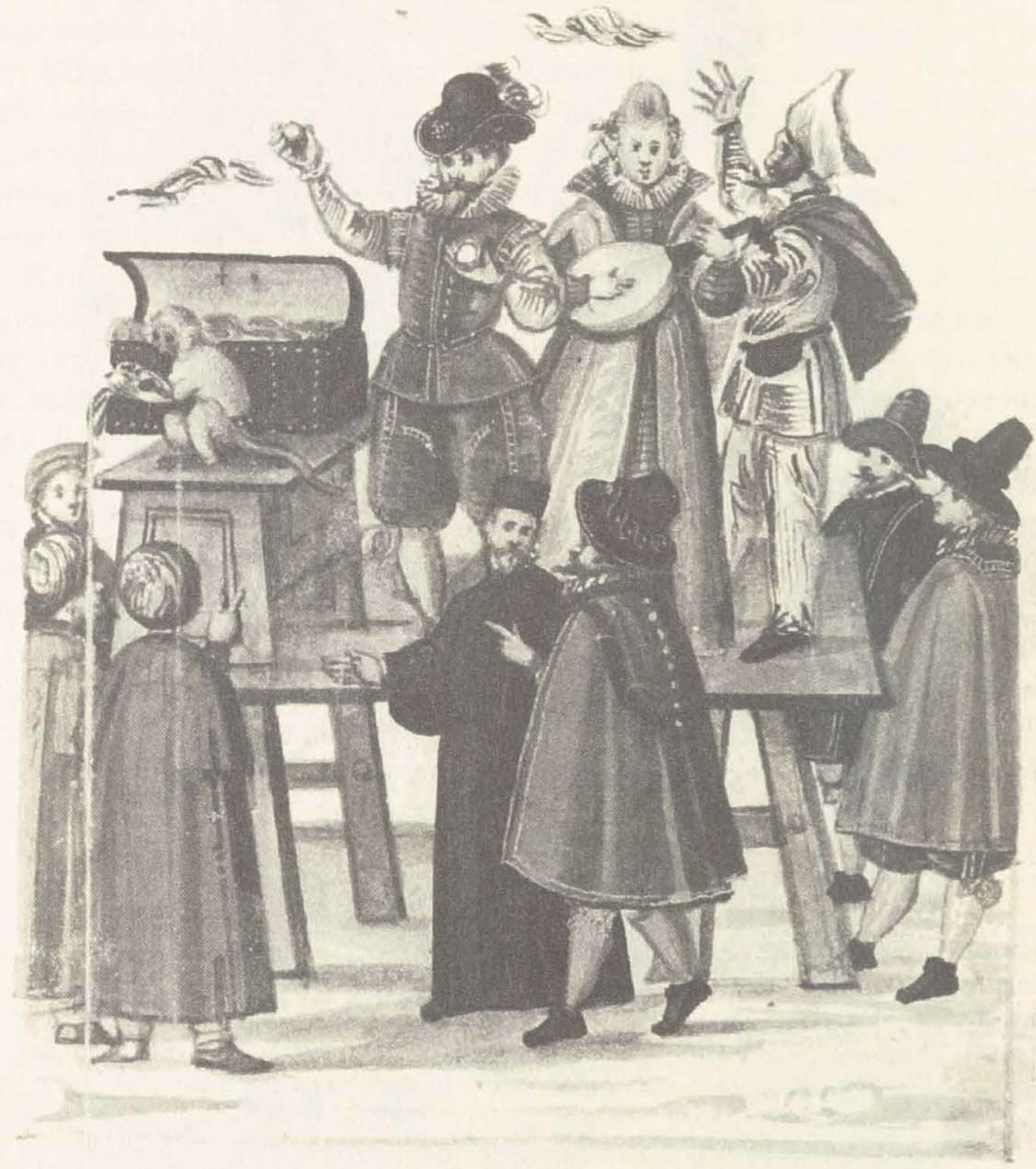

Abbildung 2: Album amicorum von Erhard Grünthaler, 1591-1612, Wien, Österreichische Nationalbibliothek (A-Wn, S.N.13.244, fol. 177v-178 , siehe auch M.A. Katritzky, Was Commedia dell'arte (wie Anm. 12), fig. 2.

Die Dame in der Mitte spielt eine Knickhalslaute, deren Saitenanzahl sich nicht bestimmen lässt. Links davon ist eine Doktor-Maske zu sehen, die kleine Büchslein in der Hand hält. Die Maske am rechten Bildrand fängt die Tücher mit den Geldstücken auf. 


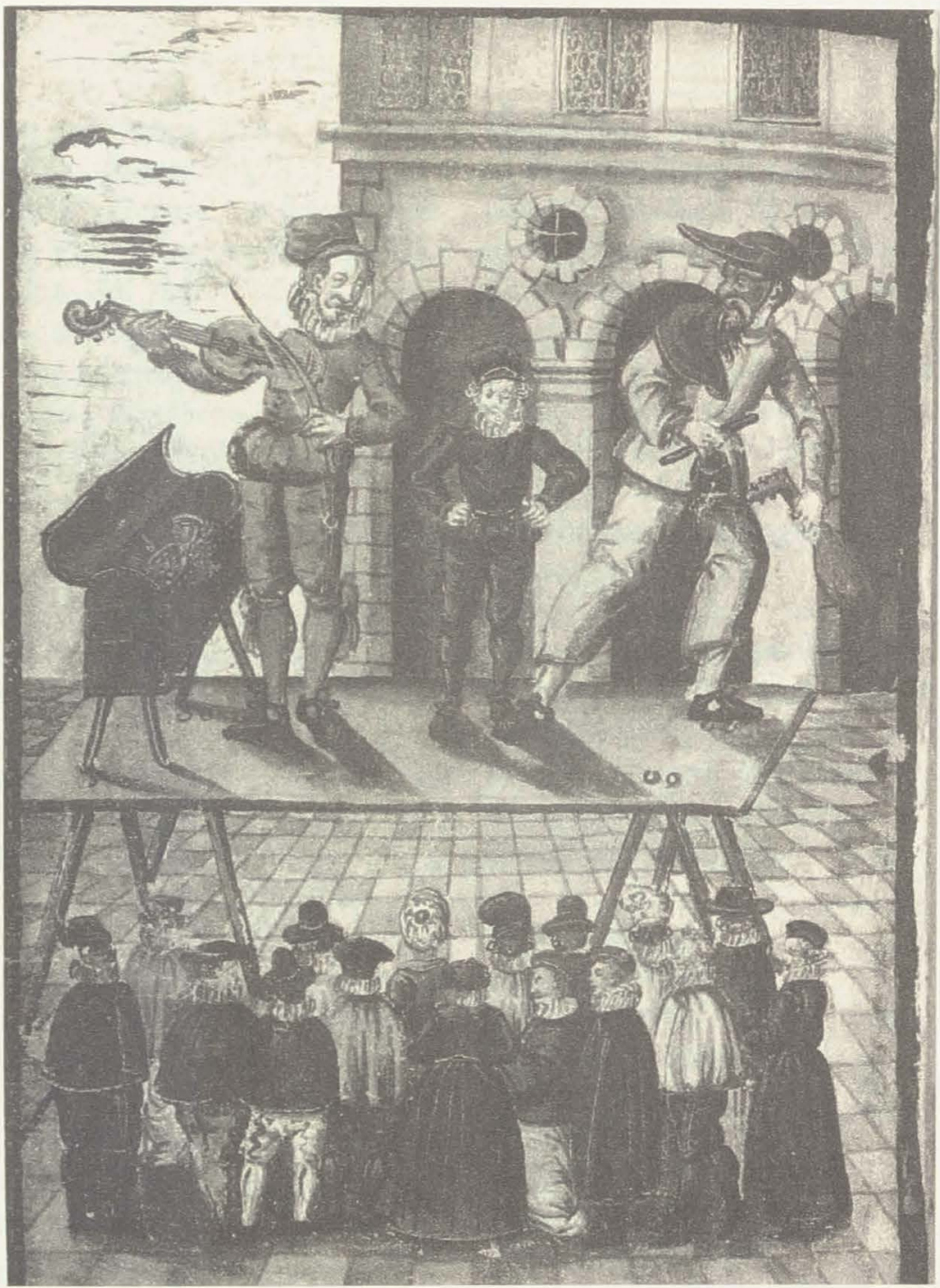

Abbildung 3: Album amicorum von Hans Eitel Neupronner, 1619-1625, London, British Library (GB-Lbl, Ms. Add.17969), fol. 7v, siehe auch M.A. Katritzky, Was Commedia dell'arte (wie Anm. 12), fig. 5 .

Am linken Bildrand ist ein Doktor neben der Medikamentenkiste zu sehen. Er musiziert mit einem konkaven Bogen auf einer 5-saitigen Viola, deren Korpus mit 2 c-förmigen und einem runden Schalloch ausgestattet, stark tailliert sowie mit einer Schnecke versehen ist. Die rechte Maske hält eine kleine Laute in der linken Hand, eventuell eine Mandora, vgl. D. Munrow, Musikinstrumente (wie Anm. 23), S. 121. Ob es sich bei dem Gegenstand in seiner rechten Hand um eine Querpfeife oder eine Flöte handelt, ist nicht genau zu erkennen. in der Mitte ist ein maskierter Gehilfe zu sehen. 